\author{
CONVEX AND FRACTAL GEOMETRY \\ BANACH CENTER PUBLICATIONS, VOLUME 84 \\ INSTITUTE OF MATHEMATICS \\ POLISH ACADEMY OF SCIENCES \\ WARSZAWA 2009
}

\title{
ON HADWIGER'S PROBLEM ON INNER PARALLEL BODIES
}

\author{
EUGENIA SAORÍN \\ Departamento de Matemáticas, Universidad de Murcia \\ Campus de Espinardo, 30100 Murcia, Spain \\ E-mail: esaorin@um.es
}

\begin{abstract}
We consider the problem of classifying the convex bodies in the 3-dimensional space depending on the differentiability of their associated quermassintegrals with respect to the oneparameter-depending family given by the inner/outer parallel bodies. It turns out that this problem is closely related to some behavior of the roots of the 3-dimensional Steiner polynomial.
\end{abstract}

1. Introduction. The Hadwiger problem. Let $\mathcal{K}^{n}$ be the set of all convex bodies, i.e., compact convex sets in the $n$-dimensional Euclidean space $\mathbf{R}^{n}$. The subset of $\mathcal{K}^{n}$ consisting of all convex bodies with non-empty interior is denoted by $\mathcal{K}_{0}^{n}$. Let $B_{n}$ be the $n$-dimensional unit ball, and $\mathbf{S}^{n-1}$ the $(n-1)$-dimensional unit sphere. The volume of a set $M \subset \mathbf{R}^{n}$, i.e., its $n$-dimensional Lebesgue measure, is denoted by $\mathrm{V}(M)$, and its boundary by bd $M$. We write $\kappa_{n}=\mathrm{V}\left(B_{n}\right)$.

For a convex body $K \in \mathcal{K}^{n}$ and a non-negative real number $\rho$ the outer parallel body of $K$ at distance $\rho$ is the Minkowski sum $K+\rho B_{n}$. On the other hand, for $0 \leq \rho \leq \mathrm{r}(K)$ the inner parallel body of $K$ at distance $\rho$ is the set

$$
K \sim \rho B_{n}=\left\{x \in \mathbf{R}^{n}: \rho B_{n}+x \subset K\right\},
$$

where $\mathrm{r}(K)$ denotes the inradius of $K$, i.e., the radius of one of the biggest balls contained in $K$ (see [2, p. 59]). Clearly if $\rho=0$ the original body $K$ is obtained. Notice that $K \sim \operatorname{r}(K) B_{n}$ is the set of incenters of $K$, usually called the kernel of $K$ and denoted by $\operatorname{ker}(K)$. The dimension of $\operatorname{ker}(K)$ is strictly less than $n$ (see [2, p. 59]). The inner parallel bodies and their properties were studied mainly by Bol [1], Dinghas [3] (see also [5] and [6]) and later by Sangwine-Yager [9].

2000 Mathematics Subject Classification: Primary 52A20, 52A39; Secondary 52A40.

Key words and phrases: Steiner polynomial, inner parallel bodies, quermassintegrals.

The author is supported by Project Phenomena in High Dimension MRTN-CT-2004-511953 of the European Community and by Dirección General de Investigación (MEC) MTM2005-08379. The paper is in final form and no version of it will be published elsewhere. 

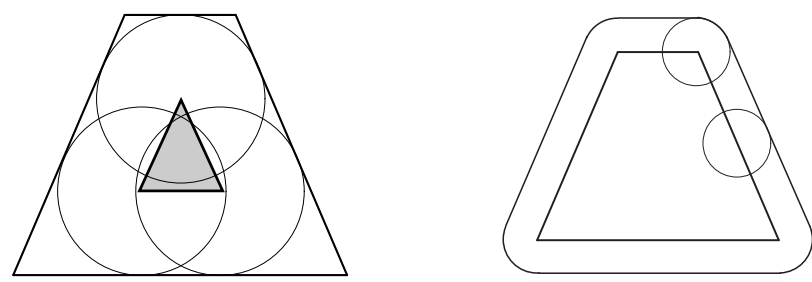

Fig. 1. The inner and the outer parallel body of a trapezoid

The full system of parallel bodies of $K$ is defined by

$$
K_{\rho}:= \begin{cases}K \sim(-\rho) B_{n} & \text { for }-\mathrm{r}(K) \leq \rho \leq 0, \\ K+\rho B_{n} & \text { for } 0 \leq \rho<\infty\end{cases}
$$

and it is a concave family, i.e., it satisfies

$$
(1-\lambda) K_{\rho}+\lambda K_{\sigma} \subset K_{(1-\lambda) \rho+\lambda \sigma}
$$

for $\lambda \in[0,1]$ and $\rho, \sigma \in[-\mathrm{r}(K), \infty)$ (see $[11, \mathrm{p} .135]$ ).

The so called Steiner formula [12] states that the volume of the outer parallel body $K+\rho B_{n}$ at distance $\rho \geq 0$ is a polynomial of degree $n$ in $\rho$,

$$
\mathrm{V}\left(K+\rho B_{n}\right)=\sum_{i=0}^{n}\left(\begin{array}{c}
n \\
i
\end{array}\right) \mathrm{W}_{i}(K) \rho^{i} .
$$

The coefficients $\mathrm{W}_{i}(K)$ are called the quermassintegrals of $K$, for which we refer to [4, s. 6.4]. In particular, $\mathrm{W}_{0}=\mathrm{V}$ is the volume (the area $\mathrm{A}$ in the planar case), $n \mathrm{~W}_{1}=\mathrm{S}$ is the surface area (the perimeter $\mathrm{p}$ in the plane), $n \mathrm{~W}_{2}=\mathrm{M}$ is the so called integral mean curvature and $\mathrm{W}_{n}=\kappa_{n}$ is the volume of the $n$-dimensional unit ball. Thus the Steiner polynomial for a 3 -dimensional convex body $K \in \mathcal{K}^{3}$ is written in terms of the volume $\mathrm{V}$, the surface area $\mathrm{S}$ and the integral mean curvature $\mathrm{M}$ of $K$ :

$$
f_{K}(\rho):=\mathrm{V}(K)+\mathrm{S}(K) \rho+\mathrm{M}(K) \rho^{2}+\frac{4}{3} \pi \rho^{3} .
$$

In [5] Hadwiger posed the problem of studying the differentiability of the coefficients of the Steiner polynomial, V, S and M, with respect to the parameter $\rho$ of the full system of parallel bodies. Next we describe this question in a precise way.

From (2) and the general Brunn-Minkowski theorem, which states that $\mathrm{V}^{1 / 3}, \mathrm{~S}^{1 / 2}$ and $\mathrm{M}$ are concave functions (see e.g. [11, p. 339]), it is easy to see that

$$
\text { i) } \left.\left.{ }^{\prime} \mathrm{V}(\rho) \geq \mathrm{V}^{\prime}(\rho) \geq \mathrm{S}(\rho), \quad \text { ii }\right)^{\prime} \mathrm{S}(\rho) \geq \mathrm{S}^{\prime}(\rho) \geq 2 \mathrm{M}(\rho), \quad \text { iii }\right)^{\prime} \mathrm{M}(\rho) \geq \mathrm{M}^{\prime}(\rho) \geq 4 \pi,
$$

where ' $F$ and $F^{\prime}$ denote, respectively, the left and right derivatives of the function $F(\rho)=$ $F\left(K_{\rho}\right)$ for fixed $K \in \mathcal{K}^{3}$ and $-\mathrm{r}(K) \leq \rho<\infty$. Hadwiger classified the convex bodies in three different classes, namely $\mathcal{R}_{\alpha}, \mathcal{R}_{\beta}$ and $\mathcal{R}_{\gamma}$, depending on wether equalities hold, respectively, in i), i) and ii), or i), ii) and iii). It is well known (see e.g. [1]) that the volume is always differentiable and $\mathrm{V}^{\prime}(\rho)=\mathrm{S}(\rho)$, which is equivalent to $\mathcal{R}_{\alpha}=\mathcal{K}^{3}$. So the question arises to characterize the convex bodies belonging to the classes $\mathcal{R}_{\beta}$ and $\mathcal{R}_{\gamma}$. 
Clearly $\mathcal{R}_{\gamma} \subset \mathcal{R}_{\beta} \subset \mathcal{R}_{\alpha}$, and all these inclusions are strict, as follows from Theorem 2 and Remark 1.

In this paper we will make first a brief survey on the known results regarding this problem (see Section 2): Theorem 1 is the classical result by Hadwiger, which provides a characterization of the values which can be the volume, surface area and integral mean curvature of some convex body in each class; Theorem 2 characterizes completely the class $\mathcal{R}_{\gamma}$; Theorem 3 and Theorem 4 state necessary conditions for a convex body to lie in $\mathcal{R}_{\beta}$, which allows to exclude families of convex bodies from this class (see Corollary 1 and Remark 1). Next, due to the close relation between the above defined classes and the behavior of the roots of the 3-dimensional Steiner polynomial, Section 3 is devoted to the study of this polynomial. Finally in Section 4 we obtain two new results (see Theorem 6 and Theorem 7) providing further necessary conditions for a convex body to lie in $\mathcal{R}_{\beta}$ but now in terms of the roots of the Steiner polynomial. Using them it will be possible to determine convex bodies not belonging to $\mathcal{R}_{\beta}$ (see Example 1 and Example 2).

2. Known results. In [5] Hadwiger gave a partial solution to this problem, in the sense of proving a characterization, not of the convex bodies, but of the triples of values $(\mathrm{V}, \mathrm{S}, \mathrm{M})$ which can be, respectively, the volume, the surface area and the integral mean curvature, of some convex body in each class:

THEOREM 1 ([5, s. 29]). Three positive real numbers V, S, M are respectively the volume, surface area and integral mean curvature of some convex body belonging to:

i) the class $\mathcal{R}_{\beta}$ if, and only if, they satisfy the inequalities

$$
\begin{aligned}
& \mathrm{V} \leq \frac{\mathrm{S}^{2}}{3 \mathrm{M}} \quad \text { and } \\
& \mathrm{V} \geq \frac{1}{24 \pi^{2}}\left[6 \pi \mathrm{MS}-\mathrm{M}^{3}-\pi\left(12-\pi^{2}\right)\left(\frac{\mathrm{M}^{2}-4 \pi \mathrm{S}}{\pi^{2}-8}\right)^{3 / 2}\right]
\end{aligned}
$$

ii) the class $\mathcal{R}_{\gamma}$ if, and only if, they satisfy the inequalities

$$
\begin{aligned}
& \mathrm{V} \leq \frac{1}{24 \pi^{2}}\left[6 \pi \mathrm{MS}-\mathrm{M}^{3}+\left(\mathrm{M}^{2}-4 \pi \mathrm{S}\right)^{3 / 2}\right] \quad \text { and } \\
& \mathrm{V} \geq \frac{1}{24 \pi^{2}}\left[6 \pi \mathrm{MS}-\mathrm{M}^{3}-\pi\left(12-\pi^{2}\right)\left(\frac{\mathrm{M}^{2}-4 \pi \mathrm{S}}{\pi^{2}-8}\right)^{3 / 2}\right] .
\end{aligned}
$$

This theorem assures that for a convex body lying in one of the classes the corresponding inequalities on $\mathrm{V}, \mathrm{S}$ and $\mathrm{M}$ hold. On the other hand, given a triple $(\mathrm{V}, \mathrm{S}, \mathrm{M})$ satisfying the inequalities in i) or ii), there will exist a convex body $K$ with $\mathrm{V}(K)=\mathrm{V}$, $\mathrm{S}(K)=\mathrm{S}$ and $\mathrm{M}(K)=\mathrm{M}$ lying in the class $\mathcal{R}_{\beta}$ or $\mathcal{R}_{\gamma}$, respectively. It does not assure however (in fact it is not true) that any convex body with those values $(\mathrm{V}, \mathrm{S}, \mathrm{M})$ for the respective magnitudes lies in the corresponding class; notice that, in general, to each triple (V, S, M) correspond many different convex bodies.

In [8] the general $n$-dimensional problem is studied, where $n$ classes appear, the so called $\mathcal{R}_{p}$ classes. There, one of the non-trivial classes is characterized, and necessary conditions for a convex body to belong to the others are stated. Particular families of 
convex bodies, e.g. polytopes and tangential-bodies, are also considered. Here we collect some of the above mentioned results in the particular case of the 3-dimensional space, which provide partial answers to the original question by Hadwiger in $\mathbf{R}^{3}$.

The following result characterizes the class $\mathcal{R}_{\gamma}$ :

TheOREM 2 ([8, Theorem 1.1]). The only convex bodies lying in $\mathcal{R}_{\gamma}$ are the outer parallel bodies of planar convex bodies, i.e.,

$$
\mathcal{R}_{\gamma}=\left\{K+\rho B_{3}: K \text { a planar convex body }\right\} .
$$

Hence, in the 3 -dimensional case, only the class $\mathcal{R}_{\beta}$ remains unknown. For this class, necessary conditions for a convex body to lie in it are stated in terms of the support function $h$ of the so called form body of $K_{\rho}$ and the set of 0 and 1-extreme normal vectors of $K_{\rho}$, which we define next.

Since our work is developed in $\mathbf{R}^{3}$, all definitions will be stated just in dimension 3 , although all of them have their analog in arbitrary dimension. As usual in the literature, we denote by $h(K, u)=\sup \{\langle x, u\rangle: x \in K\}, u \in \mathbf{R}^{3}$, the support function of $K \in \mathcal{K}^{3}$ (see e.g. [11, s. 1.7]). We write $N(K, x)$ to denote the normal cone of $K \in \mathcal{K}^{3}$ at $x \in$ bd $K$, i.e., the set of all outer normal vectors of $K$ at $x$ (with the zero vector). A vector $u \in \mathbf{S}^{2}$ is an $r$-extreme normal vector of $K, r=0,1,2$, if we cannot write $u=u_{1}+\cdots+u_{r+2}$, with $u_{i}$ linearly independent normal vectors at one and the same boundary point of $K$ (see e.g. [11, pp. 74-77]). We denote the set of $r$-extreme normal vectors of $K$ by $\mathcal{U}_{r}(K)$. Clearly $\mathcal{U}_{0}(K) \subset \mathcal{U}_{1}(K) \subset \mathcal{U}_{2}(K)$. On the other hand, the form body of a convex body $K \in \mathcal{K}_{0}^{3}$, denoted by $K^{*}$, is defined as

$$
K^{*}=\bigcap_{u \in \mathcal{U}_{0}(K)}\{x:\langle x, u\rangle \leq 1\} .
$$

The following theorem provides necessary conditions for a convex body to lie in $\mathcal{R}_{\beta}$. For the sake of brevity, from now on we write $\mathrm{r}=\mathrm{r}(K)$.

Theorem 3 ([8, Theorem 1.2]). Let $K \in \mathcal{K}^{3}$. If $K \in \mathcal{R}_{\beta} \backslash \mathcal{R}_{\alpha}$, then for all $\rho \in(-\mathrm{r}, 0]$ the following holds:

i) $h\left(K_{\rho}^{*}, u\right)=1$ for all $u \in \operatorname{cl} \mathcal{U}_{1}\left(K_{\rho}\right)$.

ii) $\operatorname{cl} \mathcal{U}_{0}\left(K_{\rho}\right)=\operatorname{cl} \mathcal{U}_{1}\left(K_{\rho}\right)$.

This theorem allows one to prove:

Corollary 1 ([8, Corollary 3.2]). There are no polytopes in $\mathcal{R}_{\beta}$.

A convex body $K \in \mathcal{K}^{3}$, with $B_{3} \subset K$, is called a $p$-tangential body of $B_{3}, p \in\{0,1,2\}$, if each $(2-p)$-extreme support plane of $K$ supports $B_{3}, p=0,1,2$. Here a supporting hyperplane is said to be $p$-extreme if its outer normal vector is a $p$-extreme direction. For characterizations and properties of $p$-tangential bodies we refer to [11, Section 2.2].

So a 0-tangential body of $B_{3}$ is just the ball itself and each $p$-tangential body is also a $q$-tangential body for $p<q \leq 2$. A 1-tangential body is usually called a cap-body, and it can be seen as the convex hull of $B_{3}$ and countably many points such that the line segment joining any pair of those points intersects the ball. A 2-tangential body will be briefly called a tangential body. 
The following result allows one again to exclude convex bodies from the class $\mathcal{R}_{\beta}$. Theorem 4 ([8, Theorem 1.3 , Theorem 1.4]). Let $K \in \mathcal{K}_{0}^{3}$.

i) $K$ is a tangential body of $B_{3}$ lying in the class $\mathcal{R}_{\beta}$ if and only if $K$ is a 1-tangential body (cap-body) of $B_{3}$.

ii) If $K \in \mathcal{R}_{\beta}$ then

$$
\mathrm{S}(K)-\mathrm{S}\left(K_{-\mathrm{r}}\right) \leq \mathrm{r}\left[2 \mathrm{M}(K)-\mathrm{rM}\left(K^{*}\right)\right]
$$

and equality holds if and only if $K$ is homothetic to a cap-body of $B_{3}$.

REMARK 1. From Theorem 4 we know that the cap-bodies of the ball lie in $\mathcal{R}_{\beta}$, and clearly they are not in $\mathcal{R}_{\gamma}$. Moreover, it assures that any 2-tangential body (which is not a cap-body) does not belong to $\mathcal{R}_{\beta}$, whereas it trivially lies in $\mathcal{R}_{\alpha}=\mathcal{K}^{3}$. It shows that all inclusions $\mathcal{R}_{\gamma} \subset \mathcal{R}_{\beta} \subset \mathcal{R}_{\alpha}$ are strict.

3. The roots of the Steiner polynomial. Regarding the Steiner polynomial (3) as a formal polynomial in the complex variable $\rho$, it is a natural question to look at its roots and to study their geometric meaning. In [7] the problem of classifying the convex bodies of the 3-dimensional Euclidean space depending on the roots of their Steiner polynomial has been solved. This classification is given in terms of relations, i.e., equalities and inequalities, between the geometric measures V, S and M. In order to state this result, we need some additional notation and definitions.

For the sake of brevity, we will denote by $\phi_{+}$and $\phi_{-}$the functionals

$$
\phi_{ \pm}(K):=\mathrm{M}(K)^{3}-6 \pi(\mathrm{M}(K) \mathrm{S}(K)-4 \pi \mathrm{V}(K)) \pm\left(\mathrm{M}(K)^{2}-4 \pi \mathrm{S}(K)\right)^{3 / 2} .
$$

On the other hand, notice that the 3 -dimensional Steiner polynomial $f_{K}(\rho)$ always has a real root. Thus, the following possibilities can appear: a triple real root, three simple real roots, a double real root and a simple one or two conjugate complex roots and the real one. In the last two cases we can distinguish two different types (see Figure 2), for which the following notation will be used: we say that the Steiner polynomial has

- double roots of TYPE 1 (complex roots of TYPE 1) if its minimum $\rho_{m}$ satisfies $f_{K}\left(\rho_{m}\right)=0\left(f_{K}\left(\rho_{m}\right)>0\right)$;

- double roots of TYPE 2 (complex roots of TYPE 2) if its maximum $\rho_{M}$ satisfies $f_{K}\left(\rho_{M}\right)=0\left(f_{K}\left(\rho_{M}\right)<0\right)$.
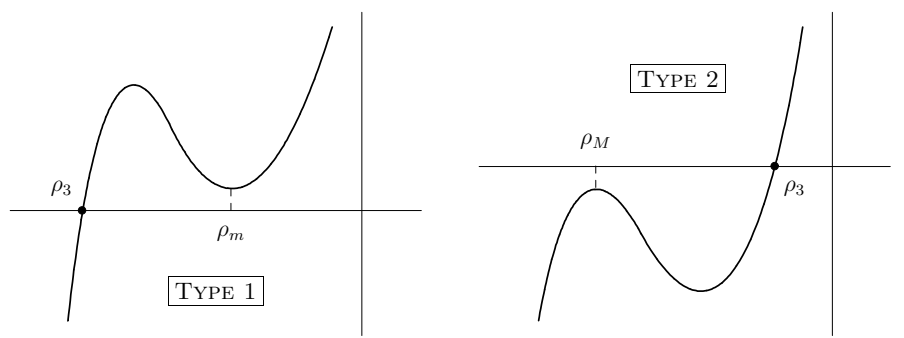

Fig. 2. The Steiner polynomial has two conjugate complex roots and a simple one. 
Using this notation, we get the following result:

Theorem 5 ([7, Theorem 1]). Let $K \in \mathcal{K}^{3}$. Then its Steiner polynomial has:

- A triple real root if and only if $K$ is a ball.

- A double real root of TYPE 1 if and only if $\phi_{-}(K)=0$.

- A double real root of TYPE 2 if and only if $\phi_{+}(K)=0$.

- Two conjugate complex roots of TYPE 1 if and only if $\phi_{-}(K)>0$.

- Two conjugate complex roots of TYPE 2 if and only if $\phi_{+}(K)<0$.

- Three simple real roots if and only if $\phi_{+}(K)>0$ and $\phi_{-}(K)<0$ simultaneously.

This theorem leads to a precise classification of the 3-dimensional convex bodies into three big (mutually disjoint) classes: the convex bodies whose Steiner polynomial has

- only real roots, either simple, double or triple (class $\Re$; in particular we write $\Re_{1}$ for double roots of TYPE 1 and $\Re_{2}$ for double roots of TYPE 2),

- complex roots of TYPE 1 ( $\left.\operatorname{class} \mathcal{C}_{1}\right)$, or

- complex roots of TYPE $2\left(\operatorname{class} \mathcal{C}_{2}\right)$.

In the particular case when $K$ is a 2-dimensional convex body in $\mathbf{R}^{3}$, its volume is $\mathrm{V}=0$, its surface area $\mathrm{S}=2 \mathrm{~A}$ and its integral mean curvature $\mathrm{M}=(\pi / 2) \mathrm{p}$. Then the Steiner polynomial of $K$ takes the form $f_{K}(\rho)=\rho\left(2 \mathrm{~A}+\pi / 2 \mathrm{p} \rho+4 / 3 \pi \rho^{2}\right)$ and it is possible to characterize the planar convex bodies of $\mathbf{R}^{3}$ depending on the algebraic type of roots that their Steiner polynomial has:

Lemma 1 ([7, Lemma 2]). If $K \subset \mathbf{R}^{3}$ is a planar convex body, its Steiner polynomial has

- three different real roots if and only if $\mathrm{p}^{2}>128 /(3 \pi) \mathrm{A}$ and $\mathrm{A} \neq 0$;

- a double real root of TYPE 1 if and only if $\mathrm{A}=0$, i.e., only if $K$ is a line segment;

- a double real root of TYPE 2 if and only if $\mathrm{p}^{2}=128 /(3 \pi) \mathrm{A}$;

- complex roots of TYPE 2 if and only if $\mathrm{p}^{2}<128 /(3 \pi) \mathrm{A}$.

- The Steiner polynomial of $K$ never has complex roots of TYPE 1.

This kind of characterization seems to be a rather useful approach to a well-known open problem in Convexity, the Blaschke problem (see [7]), although we will not consider it here (for a detailed discussion on this problem see e.g. $[5,7,10]$ ).

4. The Hadwiger problem and the Steiner polynomial. In this section we get two new results in the same line as Theorem 3 and Theorem 4: they will provide necessary conditions for a convex body to lie in $\mathcal{R}_{\beta}$, but now in terms of the type of roots of its Steiner polynomial. This will allow us to exclude new (families of) convex bodies from this class (cf. Corollary 1 and Remark 1).

Before stating the two main results of this section, we start by showing the following lemma, which will be used in the proofs of the theorems. We write $\phi_{ \pm}(\rho):=\phi_{ \pm}\left(K_{\rho}\right)$.

Lemma 2. Let $K \in \mathcal{K}^{3}$. If $K \in \mathcal{R}_{\beta} \backslash \mathcal{R}_{\gamma}$ then both $\phi_{+}(\rho)$ and $\phi_{-}(\rho)$ are strictly increasing functions in $\rho \in[-\mathrm{r}, 0]$. 
Proof. Since $K \in \mathcal{R}_{\beta}$, we have $\mathrm{V}^{\prime}(\rho)=S(\rho)$ and $\mathrm{S}^{\prime}(\rho)=2 \mathrm{M}(\rho)$ for $-\mathrm{r} \leq \rho \leq 0$. Then an easy computation gives

$$
\begin{aligned}
\phi_{ \pm}^{\prime}(\rho)=3\left[\mathrm{M}(\rho)^{2} \mathrm{M}^{\prime}(\rho)-2 \pi\left(\mathrm{M}^{\prime}(\rho) \mathrm{S}(\rho)+2 \mathrm{M}(\rho)^{2}-4 \pi \mathrm{S}(\rho)\right)\right. \\
\left.\quad \pm\left(\mathrm{M}(\rho)^{2}-4 \pi \mathrm{S}(\rho)\right)^{1 / 2}\left(\mathrm{M}(\rho) \mathrm{M}^{\prime}(\rho)-4 \pi \mathrm{M}(\rho)\right)\right] \\
=3\left[\left(\mathrm{M}(\rho)^{2}-2 \pi \mathrm{S}(\rho)\right)\left(\mathrm{M}^{\prime}(\rho)-4 \pi\right) \pm \mathrm{M}(\rho)\left(\mathrm{M}(\rho)^{2}-4 \pi \mathrm{S}(\rho)\right)^{1 / 2}\left(\mathrm{M}^{\prime}(\rho)-4 \pi\right)\right] .
\end{aligned}
$$

Since always $\mathrm{M}(K)^{2} \geq 4 \pi \mathrm{S}(K)$ for any convex body $K \in \mathcal{K}^{3}$ (see e.g. [11, p. 322]) and $\mathrm{M}^{\prime}(\rho)>4 \pi$ for $K \in \mathcal{R}_{\beta} \backslash \mathcal{R}_{\gamma}$ (see (4), iii)), we immediately get $\phi_{+}^{\prime}(\rho)>0$ for every $\rho \in[-\mathrm{r}, 0]$, which proves that $\phi_{+}(\rho)$ is strictly increasing.

In the case of $\phi_{-}(\rho)$ we have

$$
\phi_{-}^{\prime}(\rho)=3\left(\mathrm{M}^{\prime}(\rho)-4 \pi\right)\left[\left(\mathrm{M}(\rho)^{2}-2 \pi \mathrm{S}(\rho)\right)-\mathrm{M}(\rho)\left(\mathrm{M}(\rho)^{2}-4 \pi \mathrm{S}(\rho)\right)^{1 / 2}\right] .
$$

Since $\mathrm{M}(\rho)^{2}>2 \pi \mathrm{S}(\rho)$, the second term in the above product is positive if and only if

$$
\left(\mathrm{M}(\rho)^{2}-2 \pi \mathrm{S}(\rho)\right)^{2} \geq \mathrm{M}(\rho)^{2}\left(\mathrm{M}(\rho)^{2}-4 \pi \mathrm{S}(\rho)\right),
$$

which is trivially true. Moreover, $\phi_{-}^{\prime}(\rho)=0$ if and only if equality holds in the above inequality, i.e., when the surface area $\mathrm{S}(\rho)=0$ for any $\rho \in[-\mathrm{r}, 0]$. Since we have $K \notin$ $\mathcal{R}_{\gamma}$, we know that $K$ is not a planar convex body and hence $\mathrm{S}(\rho)>0$, which implies $\left(\mathrm{M}(\rho)^{2}-2 \pi \mathrm{S}(\rho)\right)^{2}>\mathrm{M}(\rho)^{2}\left(\mathrm{M}(\rho)^{2}-4 \pi \mathrm{S}(\rho)\right)$. Using again the inequality $\mathrm{M}^{\prime}(\rho)>4 \pi$ we get $\phi_{-}^{\prime}(\rho)>0$ for every $\rho \in[-\mathrm{r}, 0]$, which proves that $\phi_{-}(\rho)$ is strictly increasing.

Now we state and prove the main results in this section.

Theorem 6. Let $K \in \mathcal{K}^{3}$ with $K \in \mathcal{R}_{\beta} \backslash \mathcal{R}_{\gamma}$.

i) If $K \in \mathcal{C}_{2}$ then $K_{\rho} \in \mathcal{C}_{2}$ for every $\rho \in[-\mathrm{r}, 0]$.

ii) If $K \in \Re$ then $K_{\rho} \in \Re \cup \mathcal{C}_{1}$ for every $\rho \in[-\mathrm{r}, 0]$.

In the case $K \in \mathcal{C}_{1}$ no condition is obtained, since the Steiner polynomial of its inner parallel bodies can have any type of roots.

Proof. By Lemma 2 we know that $\phi_{+}$and $\phi_{-}$are strictly increasing functions in $\rho$, with $-\mathrm{r} \leq \rho \leq 0$, which gives $\phi_{+}(\rho) \leq \phi_{+}(0)=\phi_{+}(K)$ and $\phi_{-}(\rho) \leq \phi_{-}(0)=\phi_{-}(K)$.

i) If $K \in \mathcal{C}_{2}$ then by Theorem 5 we have $\phi_{+}(K)<0$, and hence $\phi_{+}(\rho)<0$. This shows that $K_{\rho} \in \mathcal{C}_{2}$ for every $\rho \in[-\mathrm{r}, 0]$.

ii) If $K \in \Re$ then by Theorem 5 we get $\phi_{+}(K) \geq 0$ and $\phi_{-}(K) \leq 0$; hence, in particular we have $\phi_{-}(\rho) \leq 0$. This shows that $K_{\rho} \in \Re \cup \mathcal{C}_{1}$ for every $\rho \in[-\mathrm{r}, 0]$.

We recall that $K_{-\mathrm{r}}=\operatorname{ker}(K)$ and $\operatorname{dim} K_{-\mathrm{r}} \leq 2$; hence by Lemma 1 , either $K_{-\mathrm{r}} \in \Re$ or $K_{-\mathrm{r}} \in \mathcal{C}_{2}$.

Theorem 7. Let $K \in \mathcal{K}^{3}$ with $K \in \mathcal{R}_{\beta} \backslash \mathcal{R}_{\gamma}$.

i) If $\operatorname{dim} K_{-\mathrm{r}} \leq 1$ then $K_{\rho} \in \mathcal{C}_{1}$ for every $\rho \in(-\mathrm{r}, 0]$.

ii) If $\operatorname{dim} K_{-\mathrm{r}}=2$ and $K_{-\mathrm{r}} \in \Re$ then $K_{\rho} \notin \mathcal{C}_{2} \cup \Re_{2}$ for every $\rho \in(-\mathrm{r}, 0]$.

In the case when $\operatorname{dim} K_{-\mathrm{r}}=2$ and $K_{-\mathrm{r}} \in \mathcal{C}_{2}$, no condition for all inner parallel bodies is obtained, since their Steiner polynomial can have any type of roots. We can only assure that the original body $K \in \mathcal{C}_{1} \cup \mathcal{C}_{2}$; in fact, if we suppose that $K \in \Re$ then, by Theorem 6 , part ii), we conclude that $K_{\rho} \in \Re \cup \mathcal{C}_{1}$ for all $\rho \in[-\mathrm{r}, 0]$, a contradiction since $K_{-\mathrm{r}} \in \mathcal{C}_{2}$. 
Proof. We prove first i). By Lemma 2 we know that $\phi_{+}, \phi_{-}$are strictly increasing functions in $\rho>-\mathrm{r}$, which gives $\phi_{+}(\rho)>\phi_{+}(-\mathrm{r})$ and $\phi_{-}(\rho)>\phi_{-}(-\mathrm{r})$. Since $\operatorname{dim} K_{-\mathrm{r}} \leq 1$, $\mathrm{V}(-\mathrm{r})=\mathrm{S}(-\mathrm{r})=0$ and hence

$$
\begin{aligned}
& \phi_{+}(\rho)>\phi_{+}(-\mathrm{r})=2 \mathrm{M}(-\mathrm{r})^{3}=2 \mathrm{M}\left(K_{-\mathrm{r}}\right)^{3} \geq 0, \\
& \phi_{-}(\rho)>\phi_{-}(-\mathrm{r})=\mathrm{M}(-\mathrm{r})^{3}-\mathrm{M}(-\mathrm{r})^{3}=0 .
\end{aligned}
$$

From $\phi_{+}(\rho)>0$ and by Theorem 5 we can assure that $K_{\rho} \in \Re \cup \mathcal{C}_{1}$ for all $\rho \in(-\mathrm{r}, 0]$. Moreover, since the inequality is strict, the Steiner polynomial of $K_{\rho}$ cannot have double real roots of TYPE 2 . From $\phi_{-}(\rho)>0$ and by Theorem 5 we know that $K_{\rho} \in \mathcal{C}_{1}$ for all $\rho \in(-\mathrm{r}, 0]$, i.e., the Steiner polynomial of all its inner parallel bodies has only complex roots of TyPE 1 . This shows i).

Analogously we get ii). By the strict monotonicity of the function $\phi_{+}$(in this case $\phi_{-}$ plays no role) we get $\phi_{+}(\rho)>\phi_{+}(-\mathrm{r})$. Since $\operatorname{dim} K_{-\mathrm{r}}=2$ and we assume $K_{-\mathrm{r}} \in \Re$, we know (Theorem 5) that $\phi_{+}(-\mathrm{r}) \geq 0$, which gives $\phi_{+}(\rho)>0$. This shows that $K_{\rho} \notin \mathcal{C}_{2} \cup \Re_{2}$ for every $\rho \in(-\mathrm{r}, 0]$.

We show with a couple of examples how some convex bodies can be excluded from the class $\mathcal{R}_{\beta}$ by using the previous theorems.

EXAMPLE 1. The orthogonal cylinders $K(\mathrm{r}, h)$ with circular basis of radius $\mathrm{r}$ and height $h \in[0, \infty)$ have volume $\mathrm{V}=\pi \mathrm{r}^{2} h$, surface area $\mathrm{S}=2 \pi \mathrm{r}(\mathrm{r}+h)$, and integral mean curvature $\mathrm{M}=\pi(\pi \mathrm{r}+h)$. It can be proved (see [7, Example 5]) that the Steiner polynomial $f_{K(\mathrm{r}, h)}$ can have either three real roots, or complex roots of TYPE 2, depending on the ratio between $\mathrm{r}$ and $h$. Moreover, the Steiner polynomial of just one cylinder (up to congruences) will have double real roots of TYPE 2 , the one with $h=h_{0} \approx 1.71065 \mathrm{r}$; if $h<h_{0}$, complex roots of TyPE 2 appear; if $h>h_{0}$, three simple real roots; and only in the limit case of a segment, double real roots of TYPE 1 are obtained. For instance, the archimedean cylinder (when $h=2 \mathrm{r}$ ) satisfies $K(\mathrm{r}, 2 \mathrm{r}) \in \Re \backslash\left\{\Re_{1}, \Re_{2}\right\}$.

It is easy to check that if we consider any cylinder $K(\mathrm{r}, h)$ with $h \in\left(h_{0}, 2 \mathrm{r}\right)$ then its inner parallel bodies $K(\mathrm{r}, h)_{\rho}, \rho \in[-h / 2,0]$, have

$$
\mathrm{V}=\pi(\mathrm{r}+\rho)^{2}(h+2 \rho), \quad \mathrm{S}=2 \pi(\mathrm{r}+\rho)(\mathrm{r}+h+3 \rho), \quad \mathrm{M}=\pi(\pi \mathrm{r}+h+(\pi+2) \rho),
$$

and for $\rho<-\mathrm{r}\left(h-h_{0}\right) /\left(2 \mathrm{r}-h_{0}\right)$, all the inner parallel bodies $K(\mathrm{r}, h)_{\rho} \in \mathcal{C}_{2}$, whereas we already know that $K(\mathrm{r}, h) \in \Re$. Hence by Theorem 6 we can assure that $K(\mathrm{r}, h) \notin \mathcal{R}_{\beta}$.

EXAmple 2. Theorem 7 i) allows one to exclude from $\mathcal{R}_{\beta}$ those convex bodies not lying in $\mathcal{C}_{1}$ with kernel one point. For instance, if we consider the family of cones with circular basis of radius e.g. 1 and height $h$, it is easy to check that

$$
\mathrm{V}=\frac{\pi}{3} h, \quad \mathrm{~S}=\pi\left(1+\sqrt{1+h^{2}}\right), \quad \mathrm{M}=\pi(\pi+h-\arctan h),
$$

and that they can have either real roots, complex roots of TYPE 1 or complex roots of TYPE 2. Notice that the kernel of any of these cones is a point. Hence, if $K$ is a cone with $K \notin \mathcal{C}_{1}$ (which is obtained for any $h \in[0, \bar{h} \approx 3.37108]$ ), we can assure that $K \notin \mathcal{R}{ }_{\beta}$. Notice moreover that the inner parallel bodies of any cone are homothetic copies of it. Then the Steiner polynomial $f_{K_{\rho}}$ of the inner parallel bodies will have the same type of roots as the original cone $K$, i.e., $K_{\rho} \notin \mathcal{C}_{1}$ for all $\rho \in[-\mathrm{r}, 0]$ if $K \notin \mathcal{C}_{1}$. 
Another illustrative example is provided by the truncated cones. Notice that their inner parallel bodies are cones for sufficiently small $\rho$ (see Figure 1). Hence by the previous argument for the family of cones, suitable truncated cones can be excluded also from $\mathcal{R}_{\beta}$.

REMARK 2. Notice that these results show in which cases the type of roots of the Steiner polynomial are "preserved by inner parallel bodies", in the following sense: it is known (see [7, Theorem 3]) that if $K$ is a convex body whose Steiner polynomial has a certain type of roots (simple real, double real or complex), then all the outer parallel bodies of $K$ verify that their Steiner polynomial has the same type of roots; as we can see from Theorem 6 and Theorem 7, in the case of the inner parallel bodies, this can be assured only in particular situations.

\section{References}

[1] G. Bol, Beweis einer Vermutung von H. Minkowski, Abh. Math. Sem. Univ. Hamburg 15 (1943), 37-56.

[2] T. Bonnesen and W. Fenchel, Theorie der konvexen Körper, Springer, Berlin, 1934, 1974. English translation: Theory of Convex Bodies, edited by L. Boron, C. Christenson and B. Smith, BCS Associates, Moscow, ID, 1987.

[3] A. Dinghas, Bemerkung zu einer Verschärfung der isoperimetrischen Ungleichung durch H. Hadwiger, Math. Nachr. 1 (1948), 284-286.

[4] P. M. Gruber, Convex and Discrete Geometry, Springer, Berlin, 2007.

[5] H. Hadwiger, Altes und Neues über konvexe Körper, Birkhäuser, Basel, 1955.

[6] H. Hadwiger, Vorlesungen über Inhalt, Oberfläche und Isoperimetrie, Springer, Berlin, 1957.

[7] M. A. Hernández Cifre and E. Saorín, On the roots of the Steiner polynomial of a 3dimensional convex body, Adv. Geom. 7 (2007), 275-294.

[8] M. A. Hernández Cifre and E. Saorín, On differentiability of quermassintegrals, Forum Math. (2008), to appear.

[9] J. R. Sangwine-Yager, Inner parallel bodies and geometric inequalities, Ph.D. Thesis Dissertation, University of California Davis, 1978.

[10] J. R. Sangwine-Yager, The missing boundary of the Blaschke diagram, Amer. Math. Monthly 96 (1989), 233-237.

[11] R. Schneider, Convex Bodies: The Brunn-Minkowski Theory, Cambridge University Press, Cambridge, 1993.

[12] J. Steiner, Über parallele Flächen, Monatsber. Preuss. Akad. Wiss. (1840), 114-118 [Ges. Werke, Vol. II, Reimer, Berlin, 1882, 245-308]. 
